\title{
RCS of engine inlets by a spectral iterative technique
}

\author{
Juan M. Rius, A. Lozano, A. Cardama \\ Antennas-Microwave-Radar Group, Dpt. Teoria del Senyal i \\ Comunicacions, Universitat Politècnica de Catalunya, Apdo. \\ 30002, 08080 Barcelona, Spain. Tf: 93/4016811
}

\begin{abstract}
A novel algorithm designed to compute efficiently and accurately the HF electromagnetic scattering from open-ended waveguide cavities is presented. The cavity is converted into a stepped-waveguide model so that the field spectrums are propagated, forward and backward, along each waveguide section. As boundary conditions for perfect electric conductors are applied via image theory, they are of local nature and take into account only the first order interactions between each pair of waveguide sections. Accordingly, additional forward-backward iterations must be performed if multiple interactions are to be taken into account. Finally, the RCS due to the interior irradiation is calculated by a Kirchhoff-based aperture integral. Good agreement with Method of Moments and Hybrid Modal solutions is found, as well as with experimental data, for 2-D and 3-D cavities.
\end{abstract}

\section{Introduction}

The analysis of EM scattering from open-ended waveguide cavities has received strong attention in the last years in relation with RCS reduction and target signatures. For RCS analysis of complex targets, duct structures such as jet engine intakes can often be modelled by more simple waveguide cavities.

When the dimensions are electrically small, numerical techniques such as Finite Elements Method (FEM) [1] can be applied leading to rigorous solutions. In electrically large cavities, however, HF approximations must be used due to the large number of integral equations involved. In cavities with regular uniform geometry, modal methods have proved to be efficient over a broad range of frequencies [2]. In the case of more arbitrarily shaped cavities, geometrical optics based ray-shooting [3] or Gaussian Beam (GB) [4] approaches can be used to deal with smooth non-uniform geometries, but they are limited to very high frequency problems. Recently, several hybrid schemes [5] have been reported to treat cavities which can be modelled by uniform waveguide sections connected by arbitrary transitions.

We present in this paper an alternative technique which can be considered as an evolution of previously existing spectral methods, namely the Spectral-Iterative Technique (SIT) [6] and the Spectral Incremental Procedure (SIP) [7]. The new technique provides the approximate RCS of simple cavity structures in a broad frequency range with a progressive refinement of the solution. This particular feature adapts the computational cost to the complexity of the cavity. The algorithm, however, accounts only for the interior irradiation, so that asymptotic methods should be employed to include first order edge effects.

\section{Bidimensional Algorithm}

An arbitrary 2-D cavity is plotted in Fig. 1a. To begin with, a segmentation process must be carried out as indicated in Fig. $1 \mathrm{~b}$ in order to turn the original geometry into a stepped-waveguide cavity consisting of several straight sections, each corresponding to a parallel plate waveguide piecewise.
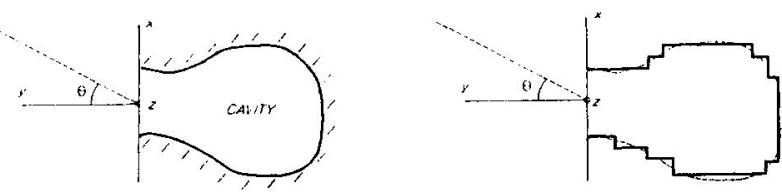

(a)

(b)
Once the modelling is completed, incident fields due to a planewave excitement are found via Kirchhoff's Approximation and the field components which will be propagated throughout $\left(\mathrm{E}^{\mathrm{Z}}\right.$ or $\mathrm{H}^{\mathrm{Z}}$ for the $\mathrm{TM}^{\mathrm{Z}}$ and $\mathrm{TE}^{\mathrm{Z}}$ cases) are sampled over the aperture. Local boundary conditions must be applied prior to the first propagation. A symmetrization procedure related with classical image theory, specular for the $\mathrm{TE}^{\mathrm{Z}}$ polarization and anti-specular for the $\mathrm{TM}^{2}$, is thus applied with respect to both conductors leading to periodical field distributions.
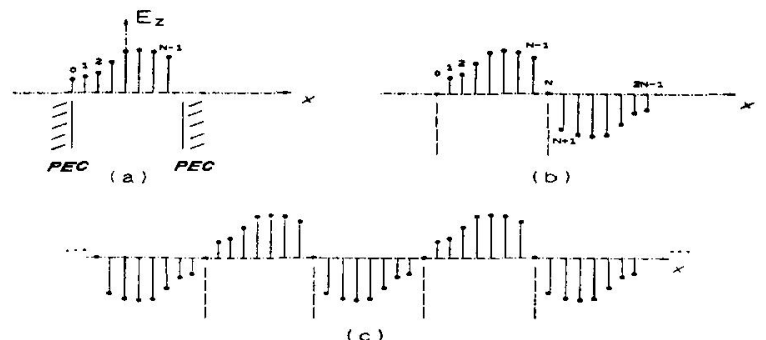

(c)

Fig. 2. Sampling and symmetrization procedure for the TMP polarization; (a) E-field sampling. (b) Anti-specular symmetrization is applied. (c) FFT-based sparial periodical extension

Plane-wave spectrums of the resulting fields, obtained via FFT, are propagated in a single step through the current waveguide section.

$$
\begin{aligned}
& \tilde{U}_{z}\left(k_{x} ; y_{0}\right)=\int U_{z}\left(x, y_{0}\right) e^{j k_{z} x} d x \\
& \tilde{U}\left(k_{x} ; y_{1}\right)=\tilde{U}\left(k_{x} ; y_{0}\right) e^{-j \sqrt{k^{2}-k_{x}^{2}}\left(y_{1}-y_{0}\right)} \\
& U_{z}(x, y)=E_{z}(x, y) \text { or } H_{z}(x, y)
\end{aligned}
$$

The fields in the spatial domain, recovered via IFFT on the final plane, include the forward contribution of the equivalent currents along the conducting walls forming the section.

$$
U_{z}\left(x, y_{1}\right)=\frac{1}{2 \pi} \int \tilde{U}_{z}\left(k_{x} ; y_{1}\right) e^{-j k_{s} x} d k_{x}
$$

In order to adjust the sampling to waveguide sections of different width, keeping samples over both conducting walls, an interpolation process must be carried out along the boundary between consecutive sections. Reflected field samples are to be stored if the following section is narrower than the previous one, while additional samples must be padded with zeros if it is wider. In the new section, symmetrization is again applied to guarantee the fulfilment of local Boundary Conditions. This procedure is repeated for every waveguide section as described in Fig. 3a.

Once the cavity end is reached, an analogous Backward Propagation procedure starts with the fields reflected in the termination end-plate. If further iterations are to be carried out, new reflected fields must be saved again as indicated in Fig. $3 \mathrm{~b}$.

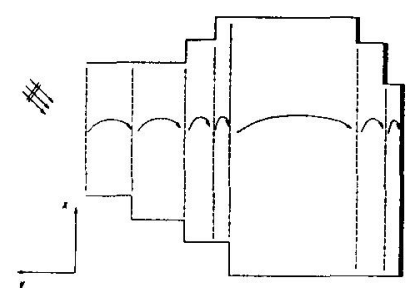

(a)

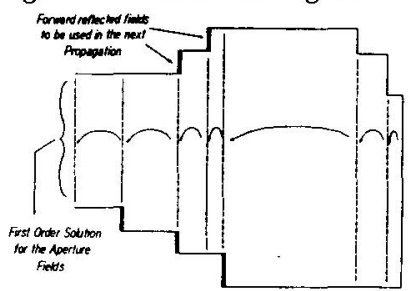

(b)
Fig. 3. (a) Forward Propagation procedure in an arbitrary 2-D cavity modelled with 7 sections. (b) Backward Propagation procedure.

A first order solution for the outwardly travelling fields is obtained after the initial iteration (forward + backward). An improved solution for the currents and the scattered fields, including higher order effects between different parts of the 
cavity, may be obtained by additional forward + backward iterations. The scheme is analogous to that of the first iteration, although in the additional forward propagations there is no incident field. The only excitement is due to the reflected fields stored in the previous backward iteration, which would represent higher order terms in a series expansion of the currents. The outwardly travelling fields obtained at each iteration must be coherently added over the aperture to form a higher order solution. As the amplitude of the successive wavefronts decreases constantly, a progressive convergence of the solution is achieved. Finally, the RCS can be calculated by an Aperture Integration based again on the Kirchhoff Approximation.

\section{Results for Bidimensional Cavities}

HF backscattering pattern of a short double-bend S-shaped cavity for the TMZ polarization is shown in Fig. 4, where comparison is established with reference solutions. Excellent agreement is observed with GB method and hybrid-modal results [4], with a single iteration.

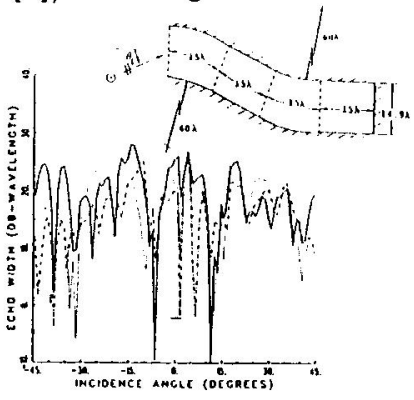

(a)

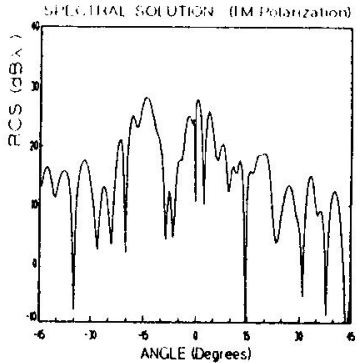

(b)
Fig. 4. RCS patterns of a shon double-bend S-shaped cavity. TM" polarization; (a) —- hybridmodal reference, . .. GB shooting method (4). (b) Spectral, I iteration, model with 22 sections.

\section{Extension to 3-D Cavities}

Modelling procedure for non-uniform rectangular cross-section geometries (Fig. 5) is analogous to that previously described for 2$D$ cavities. The cavity is converted into successive straight waveguide sections. Boundary conditions are forced again via specular or anti-specular images combined with FFT 2-D periodicity properties. Given that only two of the six vectorial components of the fields are linearly independent, only two different matrixes must be propagated by the 3-D algorithm.

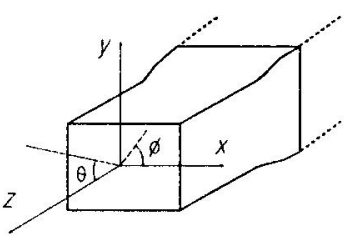

(a)

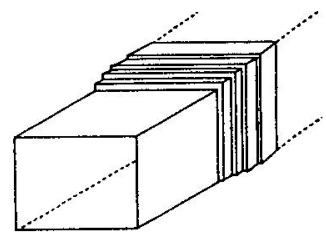

(b)
Fig. S. Modelling procedure in a 3-D caviny with rectangular cross-section; (a) Original caviry. (b) Stepped-waveguide model.

\section{Results for Tridimensional Cavities}

Shown in Fig. 6 is the comparison between numerical results generated by the Spectral Algorithm and the hybrid BIM/Modal approach, together with experimental measurements [5], for an offset rectangular waveguide cavity with two similar transition bends at $10 \mathrm{GHz}$. Good agreement is observed in the principal plane for small aspect angles until additional diffraction effects and exterior scattering come into play.

A tapered waveguide cavity composed of a sectoral section with an open-end connected to an uniform section with a planar termination has also been analyzed. Results at $10 \mathrm{GHz}$ are validated in Fig. 7 against RCS measured and modal patterns [2].

\section{Discussion and Conclusions}

The Spectral Iterative Algorithm presented in this paper has
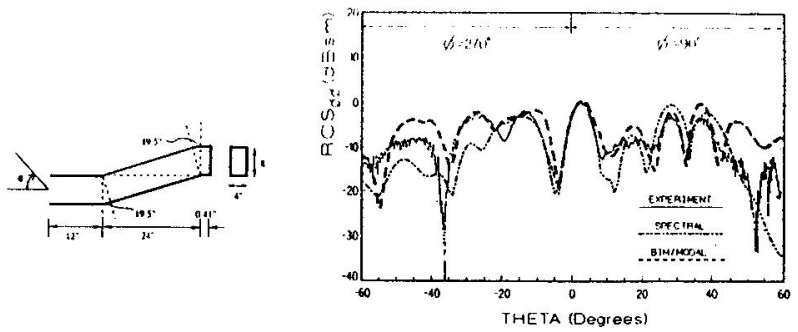

Fig. 6. Geometry of the offset rectangular cavity; Comparison berween BIM/Modal, experimenu [5]

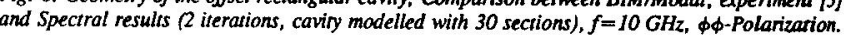
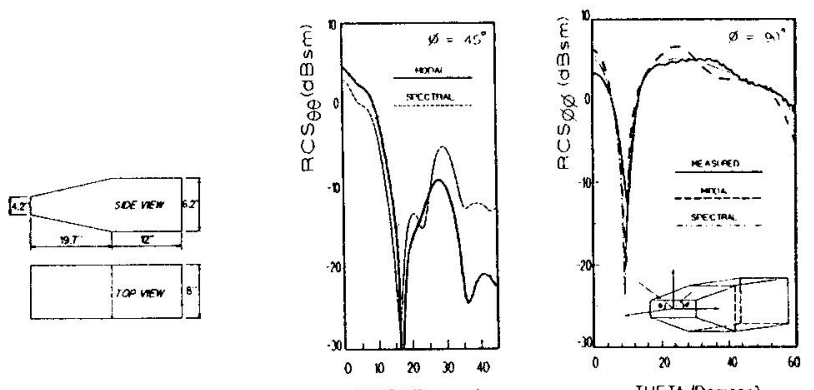

THETA Degrees)

THETA Degrees

Fig. 7. Comparison between measured, Modal [2] and Spectral ( iterations, cavity modelled with 20 sections) $R C S$ patrems, $f=10 \mathrm{GHz}$. (a) Geometry. (b) $\theta \theta$-Polarization, $\phi=45^{\circ}$ scan. (c) $\phi$ Polarization, $\phi=90^{\circ}$ scan.

shown remarkable features:

- The Algorithm requires I-D vectors in 2-D problems and 2-D matrixes in 3-D problems, leading to reduced storage and CPU necessities.

- Geometrical uniformity of the cavity under analysis is exploited: In simple bodies, the number of sections required is small whereas in complex bodies many sections must be used in the stepped model. Hence, computational cost depends on the geometrical complexity.

- The algorithm is iterative with successive refinement. The nth iteration appends to the result the fields due to multiple interaction of order $(n+1)$ between waveguide sections.

As main drawbacks we should mention that:

- The fields are distorted by the stepped shape of the cavity. In order to reduce the distortion, the modelling resolution must be increased resulting in a higher number of sections and, thus, in more FFT calculations.

- Boundary Conditions are restricted to PEC or PMC (No dielectric loadings).

- Image theory is only applicable in presence of plane conductors. Thus, the algorithm is applicable only when the cavity can be modelled by successive waveguide sections.

The computational cost relies mainly on three parameters which are closely interrelated:

- Number of sections used in the stepped model of the cavity, which depends on the geometrical complexity of the original geometry.

- Number of forward-backward iterations, which decreases with higher frequency. For electrically large cavities, a single forwardbackward iteration might be enough.

- Sampling rate. A certain oversampling factor over the Nyquist rate must be used so as to broaden the spectral window to account for some of the slower evanescent modes. The higher the sampling rate, the larger the dimension of the matrices whose FFT has to be computed. Values ranging from 64 to 512 have been utilized in the results presented.

\section{Acknowledgments}

This work has been supported by the Spanish "Comision Interministerial de Ciencia y Tecnologia" (CICYT) under the project TIC 88-288E.

\section{References}

[1] S.K. Jeng, "Aperture Admittance Matrix by Finite Element Method for Scattering from a cavity-backed Aperture", IEEE 
Antennas \& Propagat. Soc. Symp. Dig., pp. 1134-1137, Syracuse, NY, June 1988.

[2] A. Altintas, P.H. Pathak, M.C. Liang, "A selective Modal Scheme for the Analysis of EM Coupling into or Radiation from large open-ended Waveguide Cavities", IEEE Trans. Antennas \& Propagat., vol. 36, pp. 84-96, Jan. 1988.

[3] H. Ling, R. Chou, S.W. Lee, "Shooting and Bouncing Rays: Calculating the RCS of an arbitrarily shaped Cavity", IEEE Trans. Antennas \& Propagat., vol. 37, pp. 194, Feb. 89.

[4] R. Burkholder, P.H. Pathak, "Analysis of EM Penetration into and Scattering by electrically large open Waveguide Cavities using Gaussian Beam Shooting", Proc. of the IEEE, vol. 79, pp. 1401,
Oct. 91 .

[5] H. Ling, "RCS of Waveguide Cavities: A hybrid BoundaryIntegral/Modal Approach", IEEE Trans. Antennas \& Propagat., vol. 38, pp. 1413-1420, Sept. 1990.

[6] R. Kastner, R. Mittra, "A Spectral-Iteration Technique for analyzing scattering from arbitrary bodies, Part I: Cylindrical scatterers with E-Wave . . .", IEEE Trans. Antennas \& Propagat., vol. 31, May 83.

[7] G.C. Cook, A.P. Anderson, A.S. Turnbull, "Spectral Incremental Propagation (SIP) Procedure for fast Calculation of scattered Fields from conducting Bodies", Proc. of the IEE, vol. 136, pp. 28, Feb. 89. 